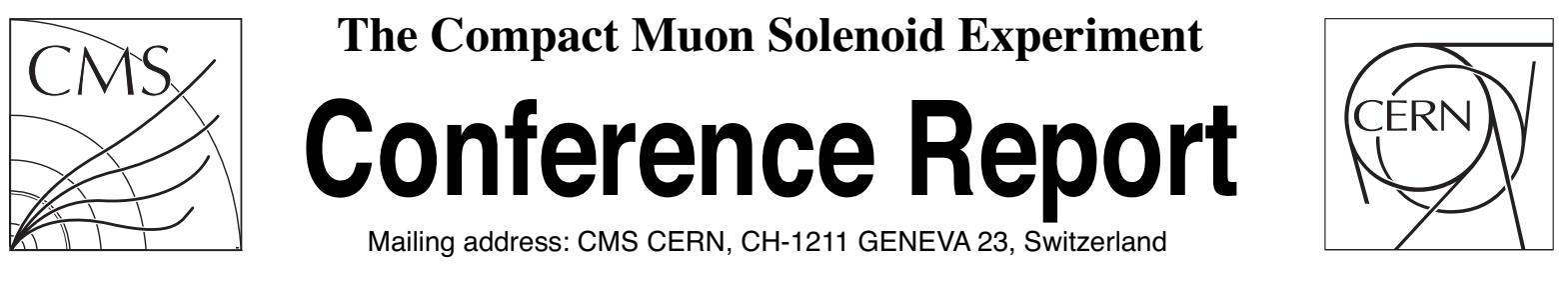

23 December 2014

\title{
STATUS REPORT ON THE CMS FORWARD MUON UPGRADE WITH LARGE-SIZE TRIPLE-GEM DETECTORS
}

Patrizia Barria for the CMS Collaboration

\begin{abstract}
For the LHC High Luminosity phase (HL-LHC) the CMS GEM Collaboration is planning to install new large size (990 x 440-220 mm2) triple-GEM detectors, equipped with a new readout system, in the forward region of the muon system (1.5 lt II lt 2.2) of the CMS detector. Combining triggering and tracking functionalities the new triple-Gas Electron Multiplier (GEM) chambers will not only improve the performance of the CMS muon trigger, but will also improve the muon identification and track reconstruction. With the addition of triple-GEM detectors the forward region of the CMS muon spectrometer will recover its originally planned redundancy. Starting from 2009 the CMS GEM Collaboration built several small and full size prototypes with different geometries, keeping improving the assembly techniques. All these prototypes have been tested in laboratories as well as with beam tests at the CERN SPS and at Fermilab. The results show that the triple-GEM detectors are a mature technology satisfying all the requirements to be used in the forward region of the CMS muon system at HL-LHC. In this contribution we will report on the status of the CMS upgrade project with GEMs and its impact on the CMS performance as also the hardware architectures and expected performance of the CMS GEM readout system.
\end{abstract}




\section{Status report on the CMS forward muon upgrade with large-size triple-GEM detectors}

D. Abbaneo ${ }^{15}$, M. Abbas $^{15}$, M. Abbrescia ${ }^{2}$, A.A. Abdelalim ${ }^{8}$, M. Abi Akl ${ }^{13}$, W. Ahmed ${ }^{8}$, W. Ahmed ${ }^{17}$, P. Altieri ${ }^{2}$, R. Aly ${ }^{8}$, C. Asawatangtrakuldee ${ }^{3}$, A. Ashfaq ${ }^{17}$, P. Aspell ${ }^{15}$, Y. Assran ${ }^{7}$, I. Awan ${ }^{17}$, S. Bally ${ }^{15}$, Y. Ban ${ }^{3}$, S. Banerjee ${ }^{19}$, P. Barria ${ }^{5 *}$, L. Benussi ${ }^{14}$, V. Bhopatkar ${ }^{22}$, Member, IEEE, S. Bianco ${ }^{14}$, J. Bos ${ }^{15}$, O. Bouhali ${ }^{13}$, S. Braibant ${ }^{4}$, S. Buontempo ${ }^{24}$, C. Calabria ${ }^{2}$, M. Caponero ${ }^{14}$, C. Caputo $^{2}$, F. Cassese ${ }^{24}$, A. Castaneda ${ }^{13}$, S. Cauwenbergh ${ }^{16}$, F.R. Cavallo ${ }^{4}$, A. Celik ${ }^{9}$, M. Choi ${ }^{31}$, K. Choi ${ }^{31}$, S. Choi ${ }^{29}$,

J. Christiansen ${ }^{15}$, A. Cimmino ${ }^{16}$, S. Colafranceschi ${ }^{15}$, A. Colaleo ${ }^{2}$, A. Conde Garcia ${ }^{15}$, M.M. Dabrowski ${ }^{15}$, G. De Lentdecker ${ }^{5}$, R. De Oliveira ${ }^{15}$, G. de Robertis ${ }^{2}$, S. Dildick ${ }^{9,16}$, B. Dorney ${ }^{15}$, W. Elmetenawee ${ }^{8}$, G. Fabrice ${ }^{27}$, M. Ferrini ${ }^{14}$, S. Ferry ${ }^{15}$, P. Giacomelli ${ }^{4}$, J. Gilmore ${ }^{9}$, L. Guiducci ${ }^{4}$, A. Gutierrez ${ }^{12}$, R.M. Hadjiiska ${ }^{28}$, A. Hassan ${ }^{8}$, J. Hauser ${ }^{21}$, K. Hoepfner ${ }^{1}$, M. Hohlmann ${ }^{22}$, Member, IEEE, H. Hoorani ${ }^{17}$, Y.G. Jeng ${ }^{18}$, T. Kamon ${ }^{9}$, P.E. Karchin ${ }^{12}$, H.S. Kim ${ }^{18}$, S. Krutelyov ${ }^{9}$, A. Kumar ${ }^{11}$, J. Lee ${ }^{31}$, T. Lenzi ${ }^{5}$, L. Litov ${ }^{28}$, F. Loddo ${ }^{2}$, T. Maerschalk ${ }^{5}$, G. Magazzu ${ }^{26}$, M. Maggi ${ }^{2}$, Y. Maghrbi ${ }^{13}$, A. Magnani ${ }^{25}$, N. Majumdar ${ }^{19}$, P.K. $\mathrm{Mal}^{6}$, K. Mandal ${ }^{6}$, A. Marchioro ${ }^{15}$, A. Marinov ${ }^{15}$, J.A. Merlin ${ }^{15}$, A.K. Mohanty ${ }^{23}$, A. Mohapatra ${ }^{22}$, S. Muhammad ${ }^{17}$, S. Mukhopadhyay ${ }^{19}$, M. Naimuddin ${ }^{11}$, S. Nuzzo ${ }^{2}$, E. Oliveri ${ }^{15}$, L.M. Pant ${ }^{23}$, P. Paolucci ${ }^{24}$, I. Park ${ }^{31}$, G. Passeggio ${ }^{24}$, B. Pavlov ${ }^{28}$, B. Philipps ${ }^{1}$, M. Phipps ${ }^{22}$, D. Piccolo ${ }^{14}$, H. Postema ${ }^{15}$, G. Pugliese ${ }^{2}$, A. Puig Baranac ${ }^{15}$, A. Radi ${ }^{7}$, R. Radogna ${ }^{2}$, G. Raffone ${ }^{14}$, S. Ramkrishna ${ }^{11}$, A. Ranieri ${ }^{2}$, C. Riccardi ${ }^{25}$, A. Rodrigues ${ }^{15}$, L. Ropelewski ${ }^{15}$, S. RoyChowdhury ${ }^{19}$, M.S. Ryu ${ }^{18}$, G. Ryu ${ }^{31}$, A. Safonov ${ }^{9}$, A. Sakharov ${ }^{10}$, S. Salva ${ }^{16}$, G. Saviano ${ }^{14}$, A. Sharma ${ }^{15}$, Senior Member, IEEE, S.K. Swain ${ }^{6}$, J.P. Talvitie ${ }^{15,20}$, C. Tamma $^{2}$, A. Tatarinov ${ }^{9}$, N. Turini ${ }^{26}$, T. Tuuva ${ }^{20}$, J. Twigger ${ }^{22}$, M. Tytgat ${ }^{16}$, Member, IEEE, I. Vai ${ }^{25}$, M. van Stenis ${ }^{15}$, R. Venditi ${ }^{2}$, E. Verhagen ${ }^{5}$, P. Verwilligen ${ }^{2}$, P. Vitulo ${ }^{25}$, D. Wang ${ }^{3}$, M. Wang ${ }^{3}$, U. Yang ${ }^{30}$, Y. Yang ${ }^{5}$, R. Yonamine ${ }^{5}$, N. Zaganidis ${ }^{16}$, F. Zenoni ${ }^{5}$, A. Zhang ${ }^{22}$

Manuscript received December 23, 2014.

${ }^{1}$ RWTH Aachen University, III Physikalisches Institut A, Aachen, Germany ${ }^{2}$ Politecnico di Bari, Universitá di Bari and INFN Sezione di Bari, Bari, Italy

${ }^{3}$ Peking University, Beijing, China

${ }^{4}$ University and INFN Bologna, Bologna, Italy

${ }^{5}$ Université Libre de Bruxelles, Brussels, Belgium

${ }^{6}$ National Institute of Science Education and Research, Bhubaneswar, India

${ }^{7}$ Academy of Scientific Research and Technology, ENHEP, Cairo, Egypt

${ }^{8}$ Helwan University \& CTP, Cairo, Egypt

${ }^{9}$ Texas A\&M University, College Station, USA

${ }^{10}$ Kyungpook National University, Daegu, Korea

${ }^{11}$ University of Delhi, Delhi, India

${ }^{12}$ Wayne State University, Detroit, USA

${ }^{13}$ Texas A\&M University at Qatar, Doha, Qatar

${ }^{14}$ Laboratori Nazionali di Frascati - INFN, Frascati, Italy

${ }^{15}$ CERN, Geneva, Switzerland

${ }^{16}$ Ghent University, Dept. of Physics and Astronomy, Ghent, Belgium

${ }^{17}$ National Center for Physics, Quaid-i-Azam University Campus, Islamabad, Pakistan

${ }^{18}$ Chonbuk National University, Jeonju, Korea

${ }^{19}$ Saha Institute of Nuclear Physics, Kolkata, India

${ }^{20}$ Lappeenranta University of Technology, Lappeenranta, Finland

${ }^{21}$ University of California, Los Angeles, USA

${ }^{22}$ Florida Institute of Technology, Melbourne, USA

${ }^{23}$ Bhabha Atomic Research Centre, Mumbai, India

${ }^{24}$ INFN Napoli, Napoli, Italy

${ }^{25}$ INFN Pavia and University of Pavia, Pavia, Italy

${ }^{26}$ INFN Sezione di Pisa, Pisa, Italy

${ }^{27}$ IRFU CEA-Saclay, Saclay, France
Abstract-For the High-Luminosity LHC (HL-LHC) phase the CMS GEM Collaboration is planning to install new largesize $\left(990 \times 220-455 \mathrm{~mm}^{2}\right)$ triple-GEM detectors, equipped with a new readout system, in the forward region of the muon system $(1.5<|\eta|<2.2)$ of the CMS detector. Combining triggering and tracking functionalities the new triple-foil Gas Electron Multiplier (GEM) chambers will improve both the performance of the CMS muon trigger and the muon reconstruction/identification in CMS experiment. The addition of triple-GEM chambers to the forward region of the CMS muon system will add a necessary layer of redundancy. Starting from 2009 the CMS GEM Collaboration has built several small and full-size prototypes with different geometries, keeping improving the assembly techniques. All these prototypes have been tested in laboratories as well as with beam tests at the CERN Super Proton Synchrotron (SPS) and at Fermi National Accelerator Laboratory. In this contribution we will report on the status of the CMS upgrade project with triple-GEM chambers and its impact on the CMS performance as well as the hardware architectures and expected capability of the CMS GEM readout system.

\footnotetext{
${ }^{28}$ Sofia University, Sofia, Bulgaria

${ }^{29}$ Korea University, Seoul, Korea

${ }^{30}$ Seoul National University, Seoul, Korea

${ }^{31}$ University of Seoul, Seoul, Korea

*Corresponding author: patrizia.barria@cern.ch.
} 


\section{INTRODUCTION}

$\mathbf{M}$ UON detection is a powerful tool for recognizing signatures of interesting physics processes over the high background rates at the Large Hadron Collider (LHC) [1]. The present muon detector system in the Compact Muon Solenoid (CMS) experiment [2] was designed to be robust, efficient, redundant, and to primarily provide muon identification, measurement of the muons transverse momentum, and correct bunch crossing assignment. Three gas detector technologies have been chosen, depending on the different radiation and magnetic environment in the CMS muon spectrometer. While the barrel region is equipped with Drift Tubes (DTs) and Resistive Plate Chambers (RPCs), on the other hand the endcap region relies on Cathode Strip Chambers (CSCs) (up to $|\eta|=2.4$ ) and Resistive Plate Chambers (RPCs) but only up to $|\eta|=1.6$. This implies that in the high- $\eta$ region $|\eta|>1.6$ the CSC is the only muon detector installed, and therefore the CMS system has limited redundancy for muon detection and recontruction. In addition, due to a rate of several tens of $\mathrm{kHz} / \mathrm{cm}^{2}$, the standard RPC technology would not sustain the harsh conditions (high integrated charge and high rate). For this reason a new technology for the $|\eta|>1.6$ region of muon system is needed to:

- Sustain a $\mathcal{O}\left(\mathrm{MHz} / \mathrm{cm}^{2}\right)$ particle rate;

- Provide good spatial resolution $\mathcal{O}(100 \mu \mathrm{m})$ for muon tracking;

- Provide good time resolution, better than $8 \mathrm{~ns}$, for muon triggering.

\section{Forward MuOn System Upgrade}

Since the beginning of the CMS data taking, all three subdetectors have been operating very well, collecting good data and accomplishing all the essential functions for muon triggering and tracking they were designed for. Actually, all the muon system performances, as well as the ones of each subdetector, do not show signs of degration and, according to the tests performed in the past, it is widely that the installed detectors should survive the whole HL-LHC phase. The tests have been performed up to a maximum of integrated charge of $100 \mathrm{mC} / \mathrm{cm}^{2}$ over 20 years of operation at the HL-LHC. Nevertheless, in order to confirm these performaces, a series of tests at the Gamma Irradiation Facility (GIF and GIF++) are planned in the next future. Altough the CMS muon system performed extremely well during the LHC Run I, it is a priority to keep this level of performance in the harsher conditions foreseen after Long Shutdown 2 (LS2) and even more after Long Shutdown 3 (LS3) in order for CMS to continue to fully exploit the performance of the LHC and to achieve its physics program. The planned strategy to ensure that the performance of the muon system will remain the same, despite the increasing luminosity and pile-up (PU), is to require an additional redundancy, such as installing additional stations in the most critical $\eta$ regions. It is precisely in this context that an intensive program of $\mathrm{R} \& \mathrm{D}$ was carried out by the CMS GEM Collaboration since 2009.

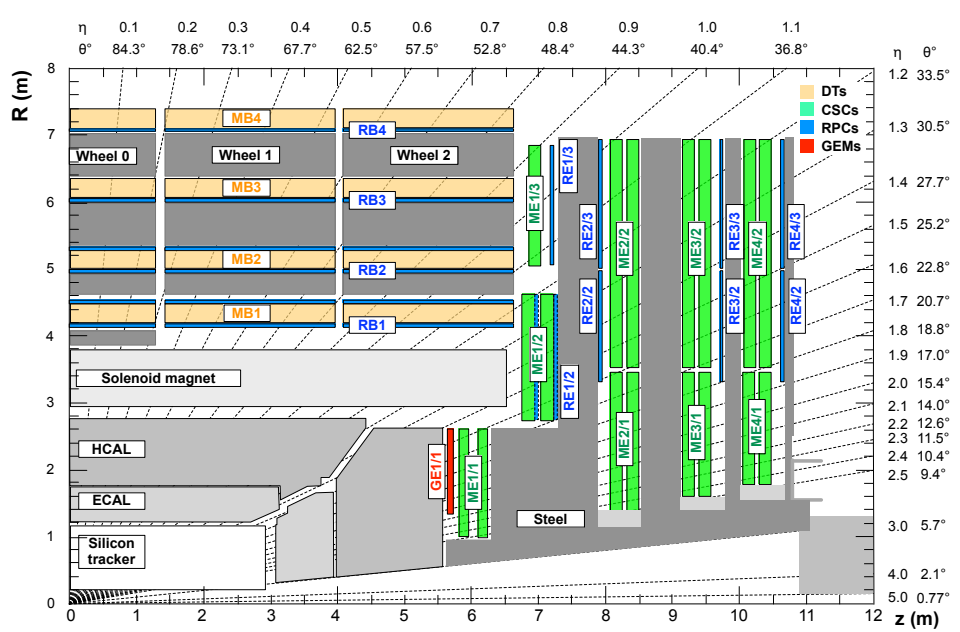

Fig. 1. Longitudinal view of a quadrant of the CMS experiment, showing the different subsystems. The location of the new forward detector proposed for Phase 2, the GE1/1 GEM station, is indicated in red.

\section{THE CMS GEM PROJECT}

Presently, the $1.6<|\eta|<2.4$ region of the CMS muon system is equipped with CSCs only, while in the initial CMS project it was designed to install in this endcap region RPC detectors as well. However, the standard bakelite RPCs suffer from the strong and increasingly hostile environment in this region and cannot handle the hit rates of a few $\mathrm{kHz} / \mathrm{cm}^{2}$, that are the ones expected in that region for the HL-LHC phase [3]. The CMS GEM Collaboration has therefore suggested the appealing Gas Electron Multiplier (GEM) technology for upgrading the muon system by proposing to instrument the nonredundant CMS high- $\eta$ region with triple-GEM detectors [4]. The most obvious locations are some existing spaces where RPCs were originally foreseen but not installed. The tripleGEMs are Micro Pattern Gaseous Detectors (MPGD) that can reach a rate capability up to $100 \mathrm{MHz} / \mathrm{cm}^{2}$ with high spatial $(100 \mu \mathrm{m})$ and time $(<5 \mathrm{~ns})$ resolution as well as detection efficiency above $98 \%$, required to improve momentum resolution for high $\mathrm{p}_{\mathrm{T}}$ muons. Figure 1 shows the possible scenario with one station equipped with triple-GEM detectors.

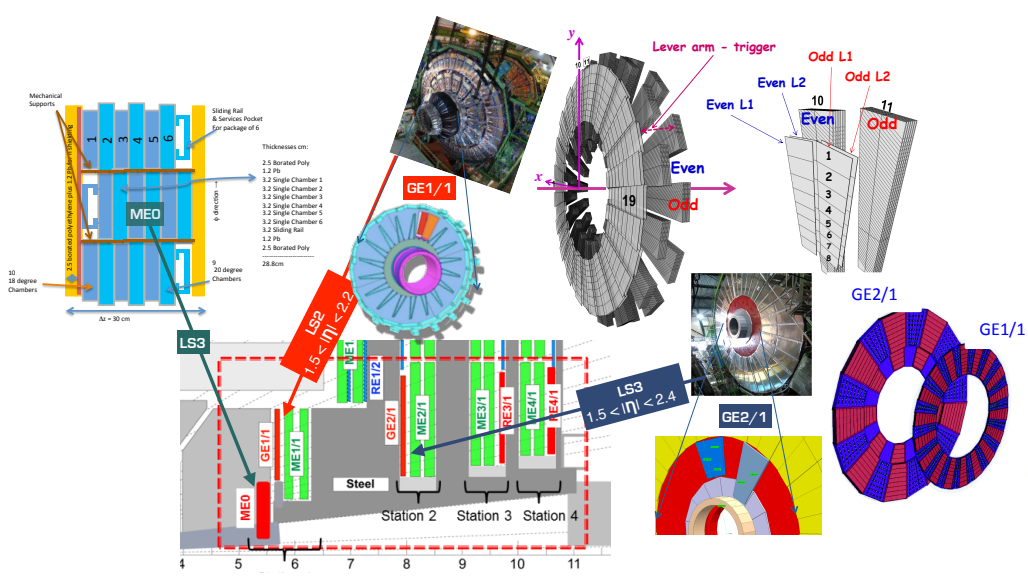

Fig. 2. Zoom of the CMS forward muon system, showing in details the new detectors proposed for LHC Phase 2. 
The guideline of the CMS GEM project foresees the installation of 36 staggered chambers per endcap just in front of the ME1/1 station (further named the GE1/1 station) during the LHC LS2. Each chamber is made of 2 back-to-back (double layered) triple-GEM chambers and spans $10^{\circ}$ in $\phi$, ensuring in this way a full coverage in $\phi$ and $1.5<|\eta|<2.1$ [5], [6]. In the present proposed schedule a second GEM station, called GE2/1, would be installed during LS3. GE2/1 would consist of 18 triple-GEM staggered chambers per endcap, each of them spanning $20^{\circ}$ in $\phi$ in the region $1.55<|\eta|<2.45$. In addiction also a muon-near tagger (ME0) composed by 6 layers of tripleGEMs, with each chamber spanning $20^{\circ}$ in $\phi$ in the region $2<|\eta|<3.5$, could be installed behind the future shortened hadron calorimeter after LS3. The future location of GE1/1, GE2/1 and ME0 within the CMS Muon system is shown in Fig. 2 while the respective proposed geometry is sketched in Fig. 3.

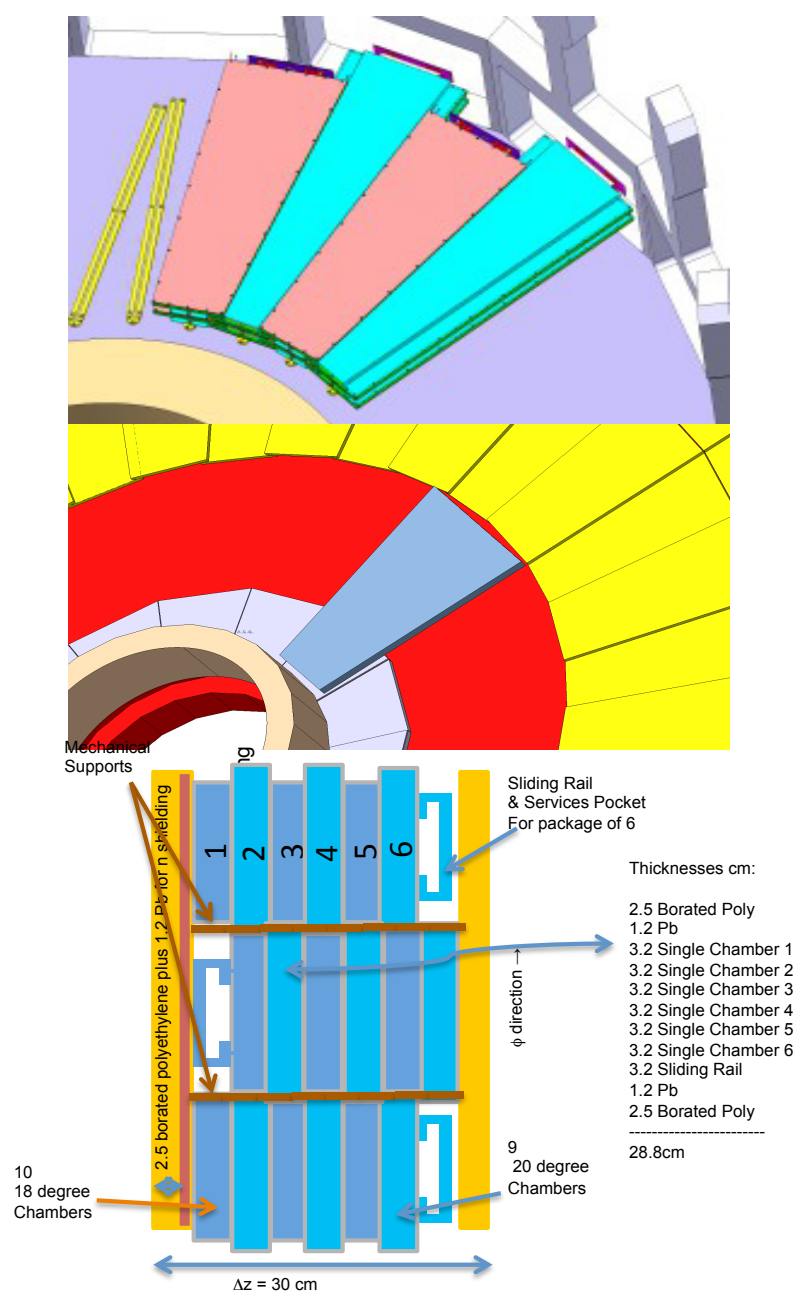

Fig. 3. Layout of the GE1/1 (top) GE2/1 (middle) and ME0 (bottom) stations that are planned to be installed in the future.

\section{CMS GEM PROTOTYPES AND R\&D PROGRESSES}

During many years of $R \& D$ at CERN, the CMS GEM Collaboration designed and produced six different GEM prototypes. The first generation of large GEM prototypes, called
GE1/1-I, introduced in 2010, consisted of eight readout sectors, with all components (frames and spacers) glued togheter. In the second generation GE1/1-II, introduced in 2011, the number of readout sectors was increased to 24 . With the third generation GE1/1-III, a new self-stretching technique, called NS2 and illustrated in Fig. 4, was introduced offering a fast GEM assembling procedure producing a flexible GEM structure. The NS2 technique offers many advantages:

- All the GEM foils are self-stretched with no spacers and no glue. The absence of spacers prevents the introduction of dead area inside the active area;

- The assembling procedure is very easy and fast (i.e. a $30 \times 30 \mathrm{~cm}^{2}$ detector can be assembled in 1 hour);

- Any detector parts are replaceable, so malfunctioning GEM detectors can be repaired quickly with low cost.

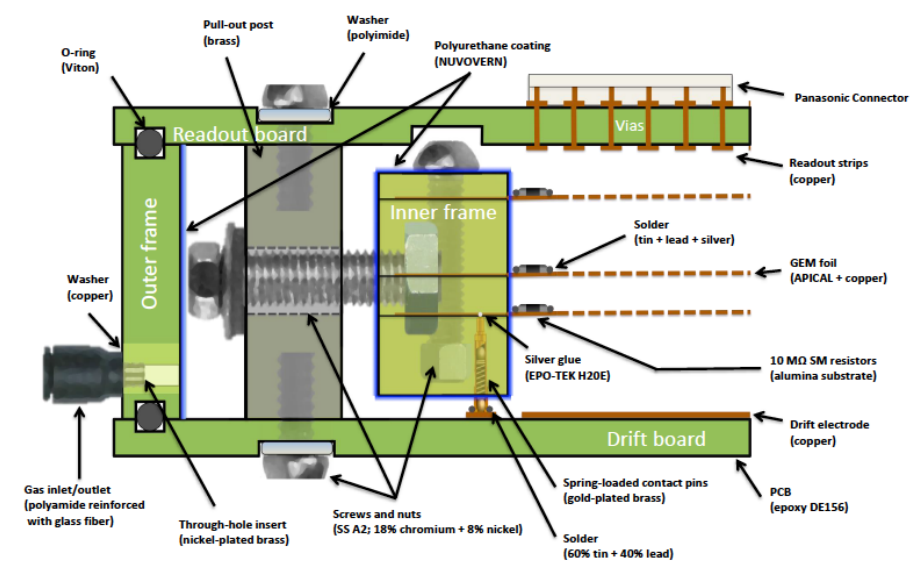

Fig. 4. Cross section of the frame region for a full-size GEM assembled with the new NS2 self-stretching technique. The GEM foils, mounted within the GE1/1 chamber, are mechanically tensioned against the brass pull-out without deforming the drift or readout boards. Also the materials of all chamber components are specified.

The GE1/1-IV was the beginning of a new era in which the prototypes began to be fully mechanically constructed and produced at several production sites. In the design of GE1/1-V we optimized the acceptance in $\eta$ and $\phi$, and built short and long type chambers. Finally the version GE1/1-VI will be the one that we will install in CMS, with more or less the same features as the GE1/1-V prototype, but also with an optimized coverage in $\mathrm{R}$.

\section{GE1/1 LAYOUT}

In the GE1/1 chamber, shown in Fig. 5, the triple-GEM detector has a trapezoidal shape with an active area of $990 \times(220-455) \mathrm{mm}^{2}$. This size is imposed by the fact that the detectors would be installed in the vacant area in the high pseudo-rapidity $(1.6<|\eta|<2.1)$ region of the CMS muon endcap system. The electrode gap configuration is $3 / 1 / 2 / 1 \mathrm{~mm}$ : it consists respectively of a drift zone, two transfer gaps and the induction gap where the electron drift will induce a signal in the readout plane of the detector. At the bottom there is the Drift Board, a printed circuit board (PCB) hosting the drift electrode and the GEM HV resistive divider. At the top there 
is instead the Readout Board divided into eight $\eta$-partition with 384 strips each oriented radially along the long side of the detector with a pitch varying from $0.6 \mathrm{~mm}$ (short side) to $1.2 \mathrm{~mm}$ (long side), while each partition is subdivided along the $\phi$-coordinate into three readout sectors, each with 128 strips or channels.

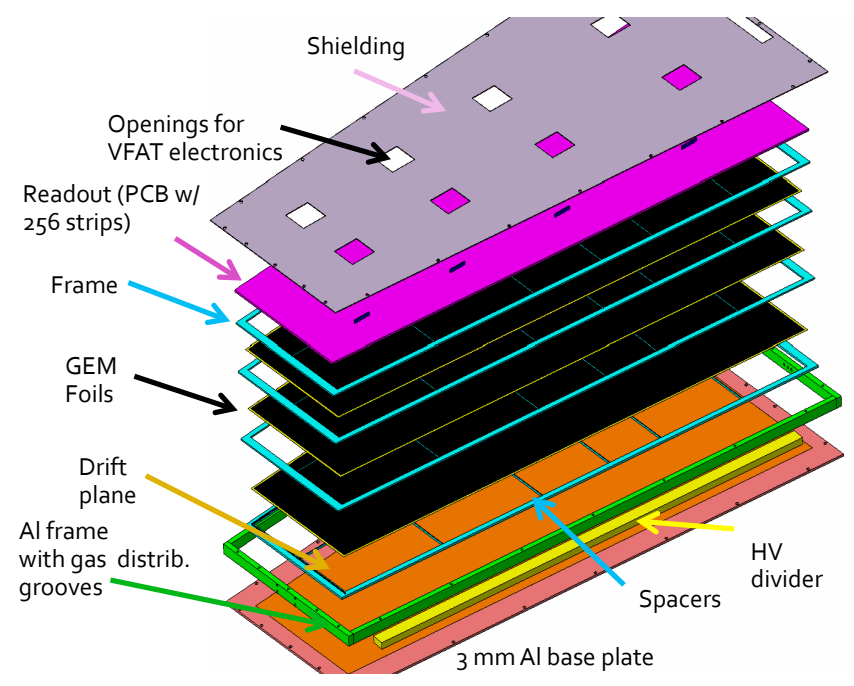

Fig. 5. Exploded 3D view of GE1/1 detector layout.

\section{GEM PROJECT ACHIEVEMENTS}

For all these years the performance of CMS triple-GEMs have been tested both in the laboratory at CERN with Xray sources as well as in many test beam campaigns with pion/muon beam at the CERN SPS [7] and in 2013 also at Fermi National Accelerator Laboratory Test Beam facility (FTBF) [8]. The detectors are mostly operated with $\mathrm{Ar} / \mathrm{CO}_{2} / \mathrm{CF}_{4}$ (45:15:40) gas mixture using as a readout either digital TURBO/VFAT2-based [9] or the Scalable Readout System (SRS) [10] with analog APV25 hybrid front-end electronics. The main results achieved can be summarized in the following table:

\begin{tabular}{|c||c|}
\hline Efficiency & $\sim 98 \%$ \\
\hline Time resolution & $\sim 4 \mathrm{~ns}$ \\
\hline Spatial resolution (binary VFAT chip) & $276 \mu \mathrm{m}$ \\
\hline Spatial resolution (analog APV chip) & $<104 \mu \mathrm{m}$ \\
\hline
\end{tabular}

Moreover, the main achievements in terms of technology and assembly are the recently introducted single-mask [11], used for the production of the foils for the large-area detectors and the new cost effective NS2 (no-spacer, no-stretch) techniques. Thanks to the use of those techniques we dramatically reduced foil production costs and allowed large-size to be manufactured and, on the other hand, we also decreased the construction time from weeks to two hours per chamber.

In addition, in view of a possible installation of the full GE1/1 station during LS2, most of the services and cabling needed are being put in place in the CMS experimental area right now during the ongoing LS1. To finalize the super-chamber layout the CMS GEM Collaboration also produced two sets of dummies GE1/1 super-chamber, one with preliminary dimensions and another one, reported on Fig. 6, alternating longshort chamber geometry. The idea was to build them with the same weight of the GE1/1 super-chambers, but without containing any detectors neither electronics, in order to verify the dimension and the insertion procedure. The first trial installation of the first set of dummies into one of the inner endcaps was succesfully done during the summer 2013 and the second one in March 2014.

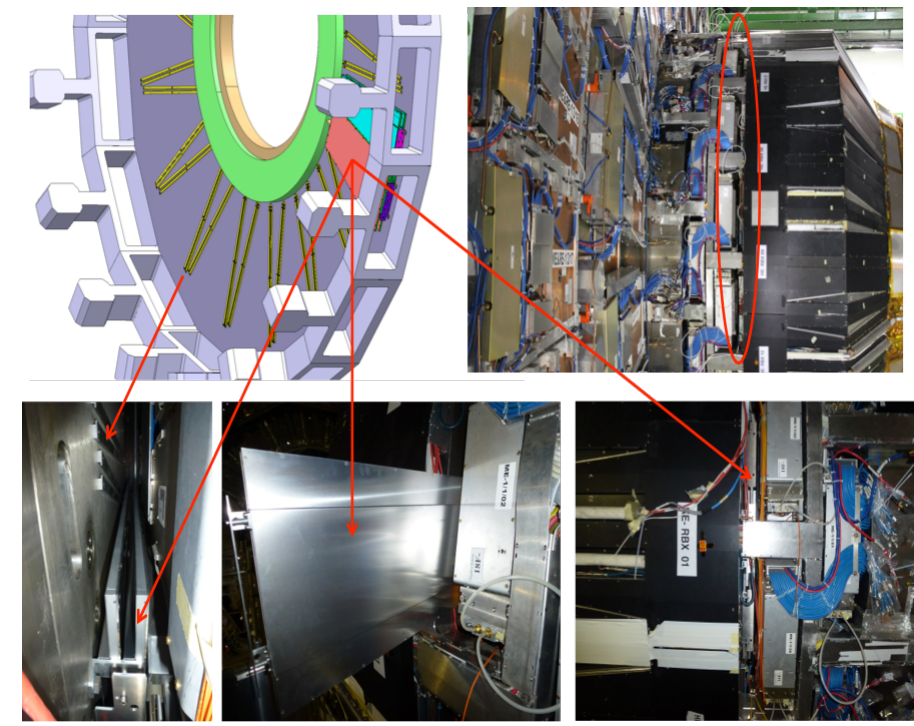

Fig. 6. GE1/1 super-chamber dummies used for trial insertion into one of the inner CMS endcap stations. The figure shows the actual situation in the nose of one of the first endcap stations; the the GE1/1 super-chambers location is indicated by the red oval shape.

\section{Forward MuOn TRIgGer CHALlenges}

\section{A. GEM-CSC Bending Angle}

The increase of the LHC beam instanteous luminosity up to $5 \times 10^{34} \mathrm{~cm}^{-2}$ will have a large impact on the muon endcap system especially in the high- $\eta$ region, which is currently suffering from the highest background rates and a non-uniform magnetic field. As a consequence of this, many more soft particles will have multiple scattering in the iron yoke, and low- $\mathrm{p}_{\mathrm{T}}$ muons can be misinterpreted as high- $\mathrm{p}_{\mathrm{T}}$ ones by the CSC Track-Finder resulting in a flattening of the trigger rate curve at high- $\mathrm{p}_{\mathrm{T}}$. Therefore the trigger rate reduction, while maintaining high efficiency, could be improved with the installation of an additional muon detector, allowing to measure the "bending angle" $\Delta \phi=\Delta \phi_{\mathrm{GEM}}-\Delta \phi_{\mathrm{CSC}}$. With the installation of an additional muon detector, such as GE1/1, in front of ME1/1 the L1 muon momentum resolution can be improved, thanks to the increased "lever arm" between both GEM and CSC stations. The GE1/1-ME1/1 bending angle will be able to effectively discriminate between soft $\left(\mathrm{p}_{\mathrm{T}} \sim 5 \mathrm{GeV}\right)$ and hard muons ( $\mathrm{p}_{\mathrm{T}}>20 \mathrm{GeV}$ ), as shown in Fig. 7(b). The even and the odd chamber pairs, mentioned in Fig. 7(b), are referred to the distance between chambers as shown in Fig. 7(a). 

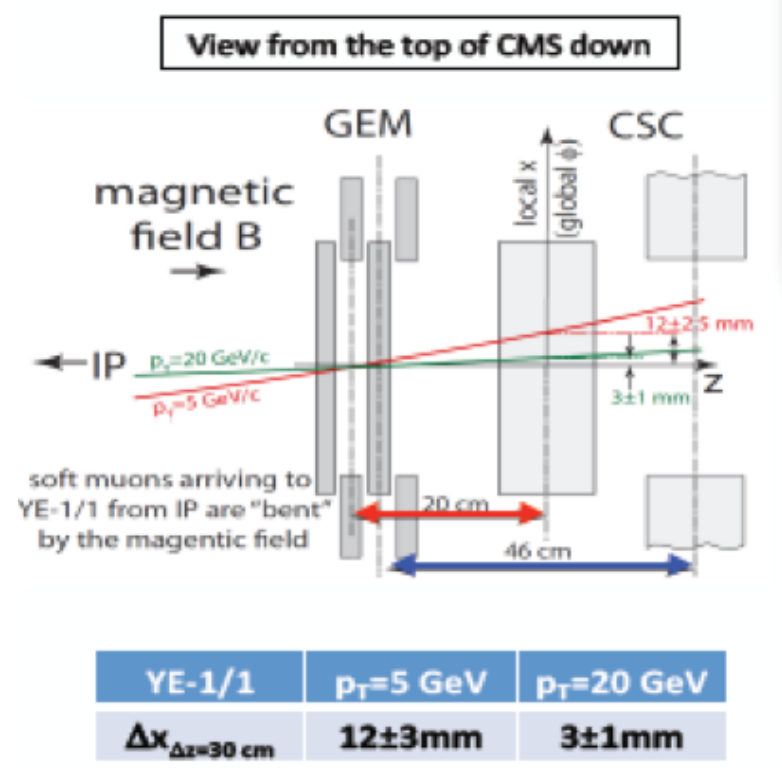

(a)
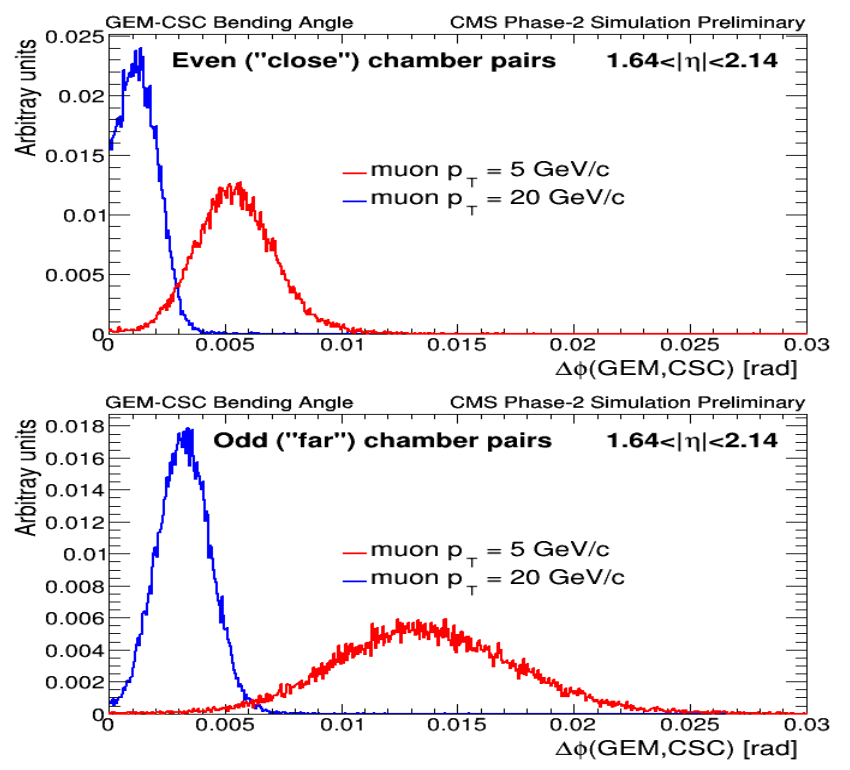

(b)

Fig. 7. Illustration of the principle of combining the GEM and CSC layers in each station to enlarge the lever arm for the bending angle measurements (a) and GEM-CSC bending angles for soft $(5 \mathrm{GeV} / \mathrm{c})$ and hard $(20 \mathrm{GeV} / \mathrm{c})$ muons respectively for even (top b) and odd (bottom b) numbered GEM chambers.

\section{B. Trigger rate}

The upgraded muon Track Finder alone should allow to decrease the $\mathrm{p}_{\mathrm{T}}$ threshold from 42 to $25 \mathrm{GeV} / \mathrm{c}$ with $9 \%$ of efficiency loss. Thanks to the combined informations from CSC and GEM, it is then possible to develop a combined local trigger to mantain or reduce the $\mathrm{p}_{\mathrm{T}}$ threshold applied at L1 to the muon candidate without efficiency loss. The better discrimination of high transverse momentum muon candidates could allow, as shown in Fig. 8, the trigger rate reduction.

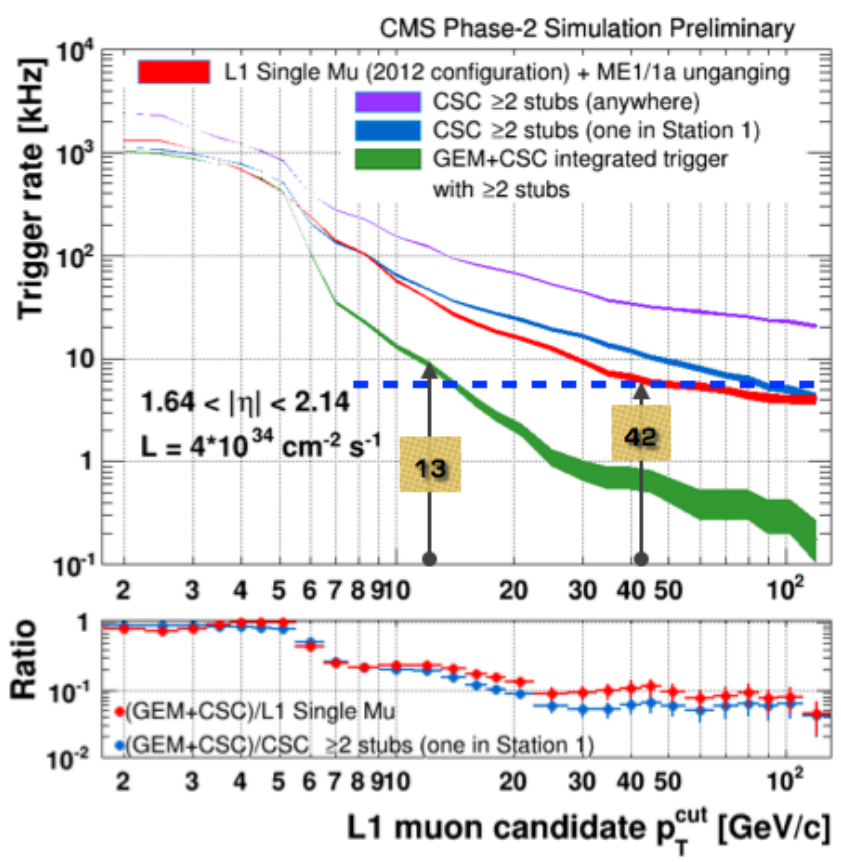

Fig. 8. Comparison of the trigger rates for the Global Muon Trigger (GMT) as a function of the L1 muon candidate transverse momentum.

Results in the region $1.6<|\eta|<2.1$, obtained with GE1/1ME1/1 combined informations, are compared with the standard CMS configuration used in 2012 (red line) modified to account for the improved ME1/1 detector electronics to be installed in LS1. Fig. 8 also shows that the GEM-CSC bending angle allows to lower $\mathrm{p}_{\mathrm{T}}$ threshold from 42 to $13 \mathrm{GeV} / \mathrm{c}$ while increasing efficiency with a consequent rate reduction of a factor 10 that can be achieved in case of a single muon trigger. For some physics channels a trigger threshold of about $15 \mathrm{GeV} / \mathrm{c}$ nearly doubles the sensitivity, that is what happens, as for example, in the $\mathrm{H} \rightarrow \tau \tau \rightarrow \mu \tau_{\text {had }}$ where yields gain in sensitivity. Lowering triggering $\mathrm{p}_{\mathrm{T}}$ from $\sim 20 \mathrm{GeV} / \mathrm{c}$ (post LS1) to $\sim 15 \mathrm{GeV} / \mathrm{c}$ and GEM+CSC yield improved background rejection result in $\sim 20 \%$ increase in acceptance as shown in Fig. 9.

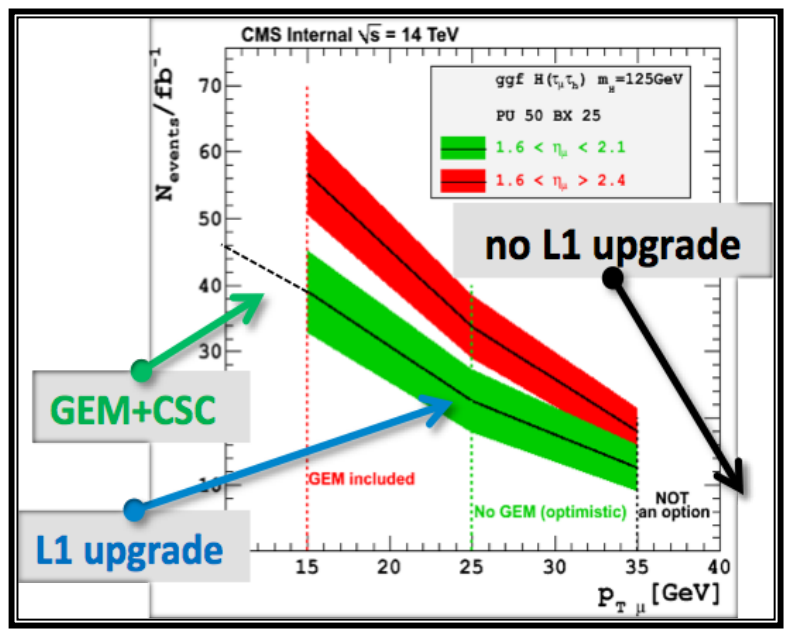

Fig. 9. Muon $\mathrm{p}_{\mathrm{T}}$ distribution in the decay channel $\mathrm{H} \rightarrow \tau \tau \rightarrow \mu \tau_{\text {had }}$ for $\tau \rightarrow \mu$. 


\section{IMPACT ON PHYSICS}

In the interesting physics channels such as $\mathrm{H} \rightarrow 4 \mu, \mathrm{Z} \rightarrow 2 \mu$ and $\mathrm{H} \rightarrow \tau \tau$ all final state muons need to be reconstructed for the full kinematic event reconstruction and $\mathrm{p}_{\mathrm{T}}$ 's are typically up to $\mathcal{O}(50 \mathrm{GeV})$. Moreover $\sim 20 \%$ of the events have at least one muon in the GE1/1 region that is the reason why it is so important to restore the redundancy in $1.6<|\eta|<2.1$ region with additional GEMs. On the other hand, it is well known how difficult this region is, because of the non-uniformity of the B field and the increasing background.

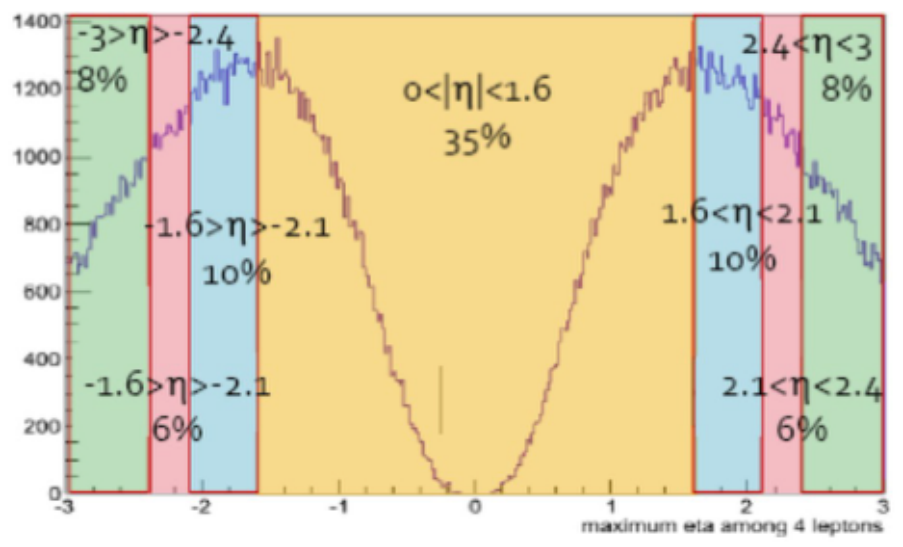

Fig. 10. Distribution of the highest $\eta$ muon from $\mathrm{H} \rightarrow 4 \mu$.

Presently the $1.6<|\eta|$ region is only instrumented with CSCs, consequently, any loss of efficiency of these chambers may result in a loss of events so this is extremely important for channel with small cross section and signal selection efficiency. Fig. 10 shows that this affects $35 \%$ of all $\mathrm{H} \rightarrow 4 \mu$.

\section{GEM PROJECT: ON GOING ACTIVITIES}

In this section is reported on the activities at the moment on going within the CMS GEM Collaboration and mainly focused on the two test beam campaigns respectively at SPS H2 (October 2014) and H4 (December 2014) beam lines.

The program is to test with two different gas mixture $\left(\mathrm{Ar} / \mathrm{CO}_{2}\right.$ (70:30) and $\left.\mathrm{Ar} / \mathrm{CO}_{2} / \mathrm{CF}_{4}(45: 15: 40)\right)$ different $\eta$ partitions of the detectors and to compare the space resolution and the efficiency between the readout sectors. Important steps are to estimate the tracking efficiency, the cluster size as well as the efficiency as a function of the current. In addition, for the first time the new GEM electronics readout system, planned for the LS2 installation, will be tested.

\section{A. October 2014 SPS H2 beam line}

During October 2014 the CMS GEM Collaboration started, at CERN Super Proton Synchroton (SPS) [12], the first of a series of two extensive test beam campaigns; the second one will take place in December 2014. Figure 11 shows the setup used during the October 2014 test beam at the CERN SPS H2 beam line, operating the triple-GEM chambers with a $\mathrm{Ar} / \mathrm{CO}_{2}$ (70:30) gas mixture and a muon beam. It consisted of a GEM tracking system, made of three $10 \times 10 \mathrm{~cm}^{2}$ honeycomb triple-GEM detectors equipped with $2 \mathrm{D}$ readout strips with

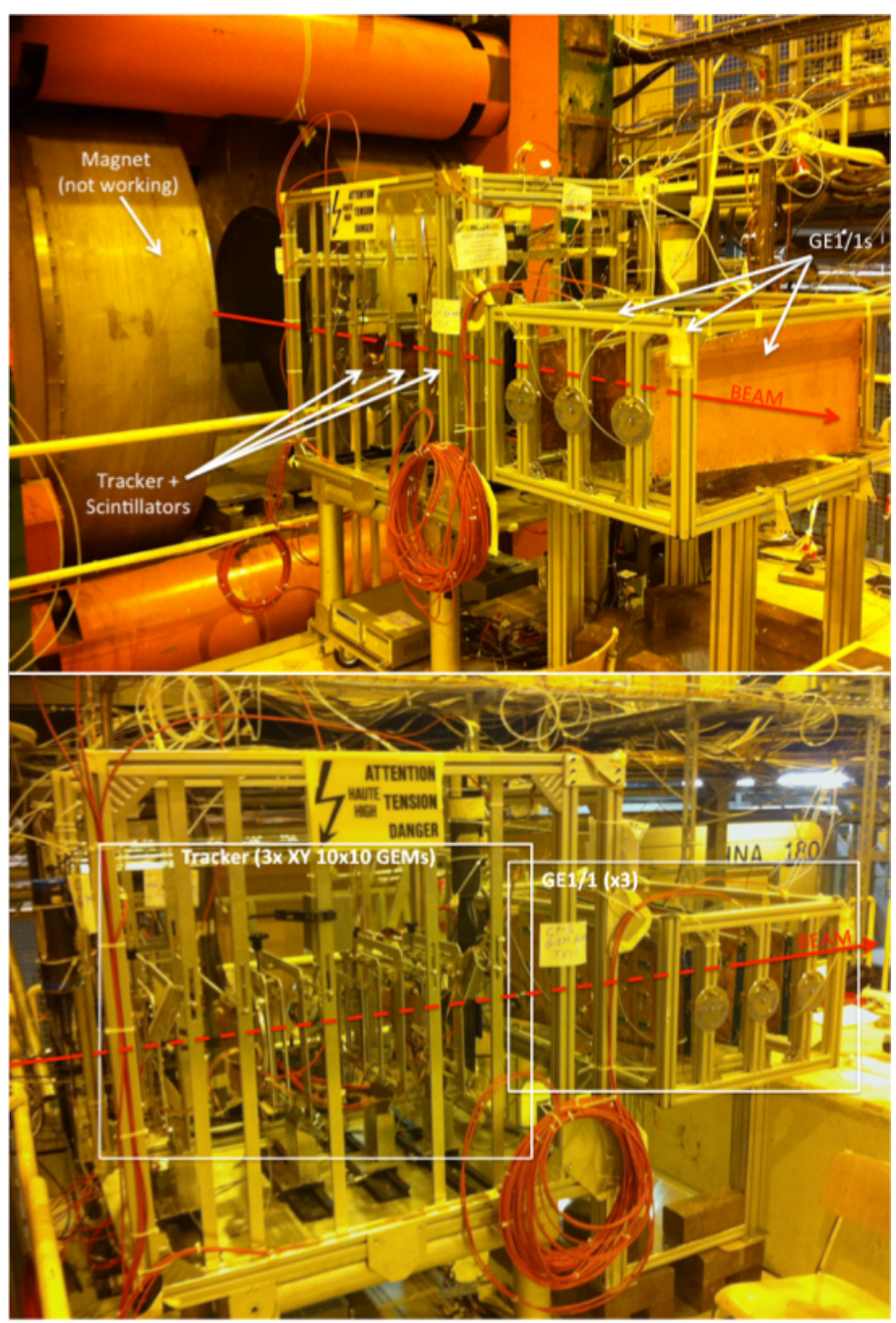

Fig. 11. Test beam setup used in the October 2014 campaign at the CERN SPS H2 beam line.

a pitch of $0.4 \mathrm{~mm}$ (2 layers under 90 degrees to provide 2D measurement), and three CMS GEM GE1/1 detectors displaced horizontally on a movable table and close to the tracking system for scanning. Both the GEM tracking system and the CMS GEM GE1/1 detectors are readout with the VFAT2-TURBO electronics. The three GE1/1 detectors used were respectively a GE1/1-IV irradiated for six months in the GIF, a GE1/1-IV not irradiated and finally a GE1/1-V. The external trigger is produced by the coincidence between the discriminated signals of 3 scintillators positioned in front and behind the GEM tracking telescope used to generate a trigger to signal the passage of a beam particle through the detector. The main goal of the first test beam has been to repeat some performance studies as well as to have a comparison in terms of efficiency between all those GE1/1 chambers. The sector irradiated was the central ones corresponding to the fourth $\eta$ partition. We performed several high voltage (HV) scans to study the efficiency as a function of the current in the $\mathrm{HV}$ divider, which is proportional to the electric fields in the GEM. The plan was mainly focused on the measurement of the spatial resolution, the time resolution, the cluster size and 
the noise levels all measurements versus the induction or drift field. We already started to analyze all data collected and we will report on the preliminary results in the coming months.

\section{B. New GEM electronics readout system tested during De- cember 2014 SPS H4 bem line}

Starting from the fall December 2014, during the second test beam campaign at the CERN SPS H4 beam line, one of purpose will be to test the new GEM electronics readout system, sketched in Fig. 12, which is planned for the LS2 installation. Each GEM detector is divided in $\eta-\phi$ sectors which are then subdivided into 128 strips, the electrodes to which the charge is induced by the passage of an ionizing particle through the detector.

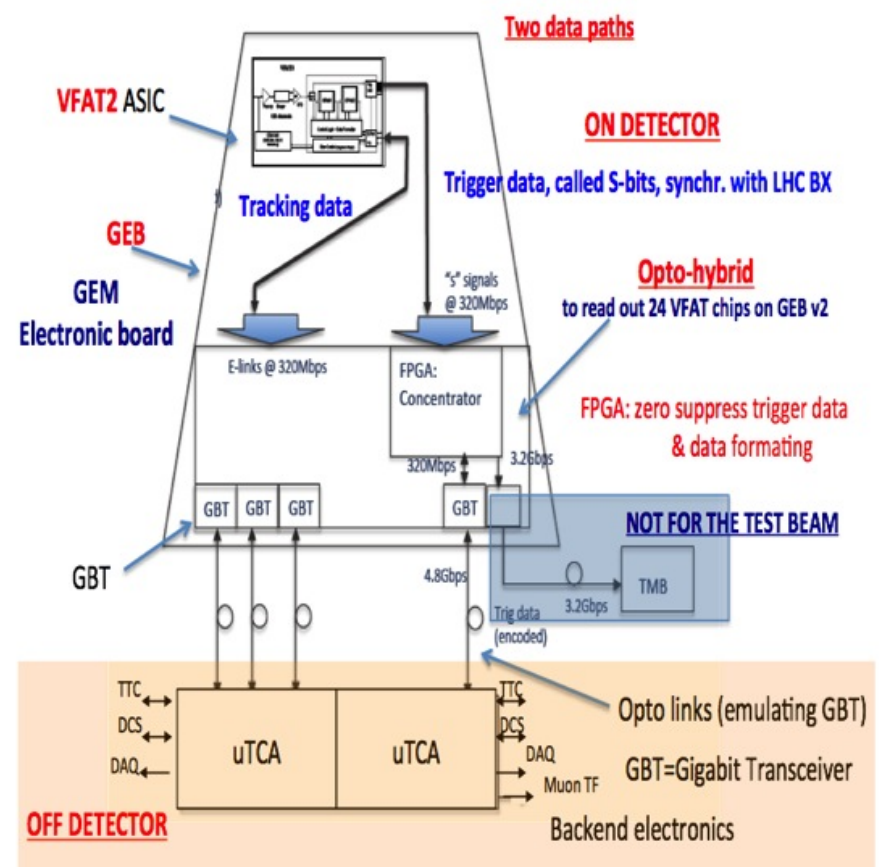

Fig. 12. Sketch of the new GEM electronics readout system.

The sketch in Fig. 12 shows the main system components for the readout of a single GEM chamber and is divided into 2 main regions, namely Off-Detector and On-Detector. In the On-Detector block there is the division of the GEM chambers in 24 sectors, as well as the 128 strips from each sector are connected to the inputs of the front-end ASIC (VFAT2) through a connector on the GEM readout board (GEB). The front-end ASICs are connected on hydrids that plug into the connectors on the Readout Board. The electrical signals (Elinks) are responsible for the control, read-out and power from the hybdrids through the GEB, covering all the entire surface. The communication to the Off-detector electronics, providing the interface from the detector, is performed through optical links via a mezzanine board, namely Opto-hybdrid, that plugs into the GEB and also contains an FPGA, GigabitTransceiver (GBT) chipset [13] and two optical connectors. The Off-detector electronics provides the interfaces from the detector (and front-end electronics) to the CMS DAQ, TTC (Trigger Timing and Control) and Trigger systems and they are implemented in the $\mu$ TCA. Two are the optical paths to and from the Opto-hybrid. The first one, running between the $\mu$ TCA crates and the Opto-hybrid, is bidirectional and is used to send setup and control signals to the front-end chips, while the return path is used for full-granularity tracking data and returning slow control data. The second one, uni-directional, takes the fixed-latency trigger data from the GEM system to the CSC Trigger Mother Board (TMB) for the L1 muon trigger.

\section{Conclusions}

The CMS GEM project started in 2009 with the idea to propose a solution for the CMS muon system upgrade for the HL-LHC phase. Presently, after 5 years of R\&D, validation of performance characteristics, quality control and assembly now the project is close to final. The project includes the installation of three new stations: GE1/1, GE2/1 in the presently vacant positions in front of $\mathrm{ME} 1 / 1$ and $\mathrm{ME} 2 / 1$, and a near-tagger, ME0. Triple-GEM detectors installation will restore the lack of redundancy in the $1.6<|\eta|<2.4$ region of the CMS muon system, at the moment only equipped with CSC, improving the muon momentum resolution measuring the bending angle and thus helping to reduce the trigger rate in the high luminosity scenarios expected in the LHC Phase-II era. In the previous test beam campaigns it has been demonstrated that the detectors exhibit a broad efficiency plateau with maximum efficiency above $98 \%$. A time resolution of $4 \mathrm{~ns}$ was obtained during drift and induction field scans. Spatial resolutions of the order of $276 \mu \mathrm{m}$ were measured with a digital readout, compatible with what one expects from the strip pitch as well as, using analog readout, spatial resolutions below $104 \mu \mathrm{m}$ were found. Finally the NS2 self-stretch technique offers not only a fast GEM assembling procedure but any detector parts are replaceable and the malfuntioning GEM detectors can be repaired quickly with low cost. These large-area GEM detectors will be a good candidate for tracking and triggering in high energy physics experiment so the CMS collaboration approved the full GE1/1 station installation possibly during the LS2.

\section{ACKNOWLEDGMENT}

We thank the RD51 Collaboration for its technical support. Also, we gratefully acknowledge support from FRS-FNRS (Belgium), FWO-Flanders (Belgium), BSF-MES (Bulgaria), BMBF (Germany), DAE (India), DST (India), INFN (Italy), Uni. Roma (Italy), NRF (Korea), QNRF (Qatar), DOE (USA), and WSU (USA).

\section{REFERENCES}

[1] L. Evans (ed.) and P. Bryant (ed.), LHC machine, JINST 3 (2008) S08001, doi:10.1088/1748-0221/3/08/S08001.

[2] CMS Collaboration, The CMS experiment at the CERN LHC, JINST 3 (2008) S08004, doi:10.1088/1748-0221/3/08/S0800.

[3] M. Tytgat et al., Construction and Performance of large-Area triple-GEM Prototypes for Future Upgrades of the CMS Forward Muon System, Proc. IEEE NSS-MIC 2011, N19-7, pp. 1019-1025.

[4] D. Abbaneo et al., GEM based detector for future upgrade of the CMS forward Muon system, Nucl.Instrum.Meth. A 718 (2013) 383-386. 
[5] The CMS GEM Collaboration, A GEM Detector System for an Upgrade of the CMS Muon Endcaps, CMS IN 2012/002.

[6] The CMS GEM Collaboration, A GEM Detector System for an Upgrade of

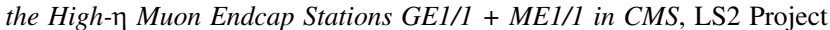
Proposal.

[7] D. Abbaneo et al., Beam Test Results for New Full-scale GEM Prototypes for a Future Upgrade of the CMS High- $\eta$ Muon System, 2012 IEEE Nucl. Sci. Symp. Conf. Rec. 1172-1176.

[8] Fermilab Test Beam Facility, http://www-ppd.fnal.gov/FTBF/

[9] P. Aspell et al., The VFAT production test platform for the TOTEM experiment, Proc. of the Topical Workshop on Electronics for Particle Physics 2008, CERN-2008-008.

[10] S. Martoiu et al., Front-end electronics for the Scalable Readout System of RD51, 2011 IEEE Nucl. Sci. Symp. Conf. Rec. 2036-2038.

[11] M. Villa et al., Progress on large area GEMs, Nucl. Instrum. Meth. A 628 (2011) 182.

[12] The Super Proton Syncrotron, http://home.web.cern.ch/about/accelerators/superproton-synchrotron.

[13] P. Vichoudis et al., The Gigabit Link Interface Board (GLIB), a flexible system for the evaluation and use of GBT-based optical links, J. Instrum. 5 (2010) C110167. 